\title{
THE GALACTIC DISTRIBUTION OF COSMIC-RAY PARTICLES
}

\author{
W. Hermsen* and J. B. G. M. Bloemen*** \\ *Laboratory for Space Research, P.O. Box 9504, 2300 RA \\ Leiden, The Netherlands \\ ** Sterrenwacht Leiden, P.O. Box 9513, 2300 RA Leiden, \\ The Netherlands
}

\section{ABSTRACT}

COS-B gamma-ray observations are compared with large-scale HI and Co surveys in different regions of the sky to derive information on the cosmic-ray density distribution throughout the Galaxy and/or a calibration of the ratio between $\mathrm{H}_{2}$ column density and integrated co Iine intensity $\left[\mathrm{N}\left(\mathrm{H}_{2}\right) / \mathrm{w}_{\mathrm{CO}}\right]$. In the region of the large complex of interstellar clouds in orion and Monoceros the observed gamma-ray emission can be explained in terms of interactions between cosmic rays distributed uniformly with the local density in this region, and the interstellar gas. In the outer galaxy a steep negative gradient of the emissivity for the 70-150 MeV energy range is found, interpreted as a steep gradient in the cosmic-ray electron density. The emissivity for the 300-5000 MeV range is found to be approximately constant (within 208) and equal to the local value out to large $(\sim 20 \mathrm{kpc})$ galacto-centric distances, interpreted as a near constancy of the nuclear component. Results sofar available for the inner galaxy indicate that this trend, a stronger gradient in the electron distribution compared to that of the nuclei, can be extrapolated into the Galaxy.

Calibration of the ratio $\mathrm{N}\left(\mathrm{H}_{2}\right) / \mathrm{W}_{c o}$ in the Orion, Monoceros region and in the first galactic quadrant give consistent values in the range $(2.5-3) 10^{20}$ molecules $\mathrm{cm}^{-2} \mathrm{k}^{-1} \mathrm{~km}^{-1} \mathrm{~s}$. The galactic centre region is found to be an anomaly: (i) the density of cosmic-ray nuclei with energies of $\sim 1 \mathrm{GeV}$ is anomalously small relative to the local value, or (ii) perhaps these cosmic rays do not efficiently penetrate the molecular clouds in the central region of the Galaxy (contrary to the case of orion) or (iii) the $\mathrm{N}\left(\mathrm{H}_{2}\right) / \mathrm{w}_{\mathrm{co}}$ ratio is anomalously low.

\section{INTRODUCTION}

The problem of the galactic distribution of cosmic rays and their origin has been under debate for over half a century. In fact, it is now precisely 50 years ago that Baade and $z w i c k y$ /1/ first proposed that supernova explosions could provide the energy for accelerating cosmic rays. In this issue one can read that presently supernova explosions are only one of several candidates for the origin of the high-energy cosmic-ray electrons and nuclei.

Important information in the search for the origin of cosmic rays would be detailed knowledge of their galactic distribution. However, the latter can only be measured indirectly, i.e. the large-scale galactic synchrotron emissivity reflects the distributions of cosmic-ray electrons and magnetic fields in the Galaxy (e.g. $/ 2 /)$ and the large-scale galactic gamma-ray ( $₹ 250 \mathrm{MeV}$ ) distribution is predominantly a tracer of cosmic-ray electrons and nuclei and the interstellar gas $(e . g . / 3,4 /)$.

First attempts to construct a consistent model using galactic distributions of the nonthermal radio emission, the gama-ray emissivity and the interstellar gas have been presented by Cassé, Cesarsky and paul /5,6/ and Higdon /7/. The main problems these authors encountered in 1976-1978 were the large uncertainties in the molecular-hydrogen distribution (two-dimensional co surveys over large areas of the sky, used to trace molecular hydrogen, had just been started) and the low counting statistics in the gamma-ray data. At the moment, the experimental situation in the fields of $\mathrm{mm}$ - and gamma-ray astronomy has notably improved. For example, extended regions of the sky have been completely sampled in co and the longer lifetime in orbit of the cos-B $/ 11 /$ gamma-ray telescope $/ 12 /$, compared to that of the SAS-2 instrument $/ 13 /$, resulted in an improvement in statistics by more than an order of magnitude. The observations become now sufficiently good to aim in the near future for a self-consistent model for the large-scale galactic magnetic-field, cosmic-ray, and total mass distributions using the large-scale synchrotron emissivity, gamma-ray emissivity, HI and Co distributions. In this paper we will limit ourselves to the recent contributions by the caravane Collaboration for the $\cos -\mathrm{B}$ mission to this general problem. Namely, the distribution of cosmic-ray particles derived from correlation studies between the improved radio/mm data and gamma-ray observations. 
COSMIC RAYS, GAS AND GAMMA RAYS

The diffuse component of galactic gama radiation in the energy band discussed in this paper $(E>70 \mathrm{MeV}$ ) has long been interpreted to be mainly the result of the interaction of cosmicray electrons (via bremsstrahlung, the dominant process at low energies, $E \leqslant 150 \mathrm{MeV}$ ) and cosmic-ray nuclei (via $\pi^{\circ}$-decay, dominant at higher energies, $E>300 \mathrm{MeV}$ ) with the interstellar gas. The produced gamma-ray intensity, $\mathrm{I}_{\gamma}$, can be formulated as follows:

$$
I_{Y}=\left(q_{Y} / 4 \pi\right)\left[\mathrm{N}(\mathrm{HI})+2 \mathrm{~N}\left(\mathrm{H}_{2}\right)\right] \text {. }
$$

in which $q_{\gamma}$ is the ganma-ray production rate per $\mathrm{H}$ atom, proportional to the cosmic-ray density, and $\mathrm{N}$ is the column density of hydrogen atoms ( $\mathrm{HI}$ ) and molecules $\left(\mathrm{H}_{2}\right)$. Therefore, gamma radiation is a tracer of the product of the cosmic-ray density and the interstellar gas density, integrated along the line of sight. Gamma-ray measurements can provide a diagnostic of the cosmic-ray density in regions where the interstellar gas is well traced at other wavelengchs, as well as a diagnostic of the total gas content in cases where the cosmic-ray density can be assumed to be equal to the value for the solar neighbourhood (local value, $s$, $\mathrm{kpc})$. The latter has been verified at intermediate latitudes $b=10^{\circ}-20^{\circ}$, using galaxy counts as a total-gas tracer for the local interstellar medium (see e.g. $/ 14,15,16 /$ ). A good correlation was found between gamma-ray intensities and total-gas column densities.

of the two dominant constituents of the interstellar gas, HI and $\mathrm{H}_{2}$, the HI distribution has been mapped on a large scale, using its characteristc $21-\mathrm{cm}$ line, and with sufficient accuracy for the correlation study with the available gamma-ray data. However, the conversion of observed $\mathrm{CO}$ emission to $\mathrm{H}_{2}$ column densities is ambiguous (see e.g. /17/) . In fact, gamma-ray astronomy can provide new insight into this calibration problem.

In the actual comparison between measured gamma-ray intensities and atomic- and molecularhydrogen column densities equation (1) becomes :

$$
I_{\gamma}=\left(q_{\gamma} / 4 \pi\right)\left[N(H I)+2 x W_{C O}\right]+I_{b} \text {, }
$$

where $\mathrm{w}_{\mathrm{CO}}$ is the integrated temperature of the ${ }^{12} \mathrm{CO} 2.6-\mathrm{mm}$ 1ine (which is best mapped at the moment) and $\mathrm{x}=\mathrm{N}\left(\mathrm{H}_{2}\right) / \mathrm{W}_{\mathrm{CO}}$ is the $\mathrm{N}\left(\mathrm{H}_{2}\right)-$ to- $\mathrm{W}_{\mathrm{Co}}$ conversion factor, if all the gas is pervaded by the same cosmic-ray flux. $I_{b}$ is the background level, which is mainly instrumental. $\mathrm{N}(\mathrm{HI})$ and $\mathrm{W}_{\mathrm{co}}$ have to be convolved with the energy-dependent point-spread function of the cos-B instrument /18/ before making comparisons with the gamma-ray intensities. If the convolved distributions of $\mathrm{N}(\mathrm{HI})$ and $\mathrm{w}_{\mathrm{CO}}$ show significant (and mutually different) structure, a multiple-linear regression method can be used to determine $q_{\gamma} x$ and $I_{b}$. To obtain most of the results in this paper a maximum likelihood method, similar to that used in $/ 14 /$, was applied on $1^{\circ} \times 1^{\circ}$ bins. The ganma-ray data used are those described in $/ 12 /$, supplemented by later observations. HI column densities are determined from the Berkeley $21-\mathrm{cm}$ line surveys $/ 19,20 /$ and a southern-hemisphere survey of the galactic plane by strong and coworkers $/ 21 \%$ The $\mathrm{CO}$ surveys used are from the Goddard Institute for Space Studies and Columbia University $/ 8,9,10 /$ or those from Bania covering the galactic-centre region $/ 22,23,24 /$.

\section{COSMIC RAYS IN THE ORION-MONOCEROS REGION}

The large complex of clouds in Orion and Monoceros, is the most extensively studied in $C O$ at medium latitudes $18,9 /$; the coverage is shown in Figure $1 \mathrm{~b}$. All experimental data for this region of the sky is available (Figure $1 a, c, d$ ) which enables a correlation study following equation (2). In addition, it is away from the intense ridge of gamma radiation along the galactic plane. Therefore, it is possible to verify in detail whether the local cosmic-ray density can explain the measured gamma rays originating in the HI mass as well as those originating in the $\mathrm{H}_{2}$ clouds.

Previous studies of one cos-B observation of the orion complex /25,26/ did reveal the eloud complex in gamma rays and did yield a total mass of the complex in agreement with independent radio-astronomical evaluations. Now all $\cos -B$ observation periods, each of at least one month duration, covering the region shown in Figure 1 can be used (seven observation periods contribute significantly). To perform a three-parameter fit following equation (2), the 300-5000 $\mathrm{MeV}$ range was selected to exploit the better angular resolution of COS-B at high ganma-ray energies (FMWH $\cong 1.5^{\circ}$, HPBW $\cong 3.4^{\circ}$ ). With these angular parameters the structures in the gammaray map due to the three constituents, $\mathrm{HI}, \mathrm{H}_{2}$ and background, remain significantly different.

The derived value for the background level is consistent with other analyses of the same $\cos -B$ data base $(e . g . / 15 /)$ and $q(300-5000 \mathrm{MeV}) / 4 \pi=(0.52 \pm 0.13) \times 10^{-26} \mathrm{ph} \mathrm{H}^{\mathrm{H}} \mathrm{at} \mathrm{s}^{-1} \mathrm{st}^{-1}$ agrees with the average local values determined by Strong et al. $/ 15 /\left[(0.59 \pm 0.15) \times 10^{-26}\right.$ ph $\mathrm{H}$ at $\left.{ }^{-1} \mathbf{s}^{-1} \mathrm{sr}^{-1}\right]$ using galaxy-count data at intermediate latitudes, and in the Bloemen et al. (/27) and summarized below) paper $(0.53 \pm 0.14) \mathrm{x}^{-26} \mathrm{ph} \mathrm{H}$ at $\mathrm{t}^{-1} \mathrm{~s}^{-1} \mathrm{sr}^{-1}$ from the radial distribution of the ganma-ray emissivity in the outer galaxy. Furthermore, the derived gammaray emissivity is consistent with values calculated theoretically for cosmic-ray-matter interactions $(e . g \cdot / 28,29 /)$. The third free parameter derived from the fit is found to be 

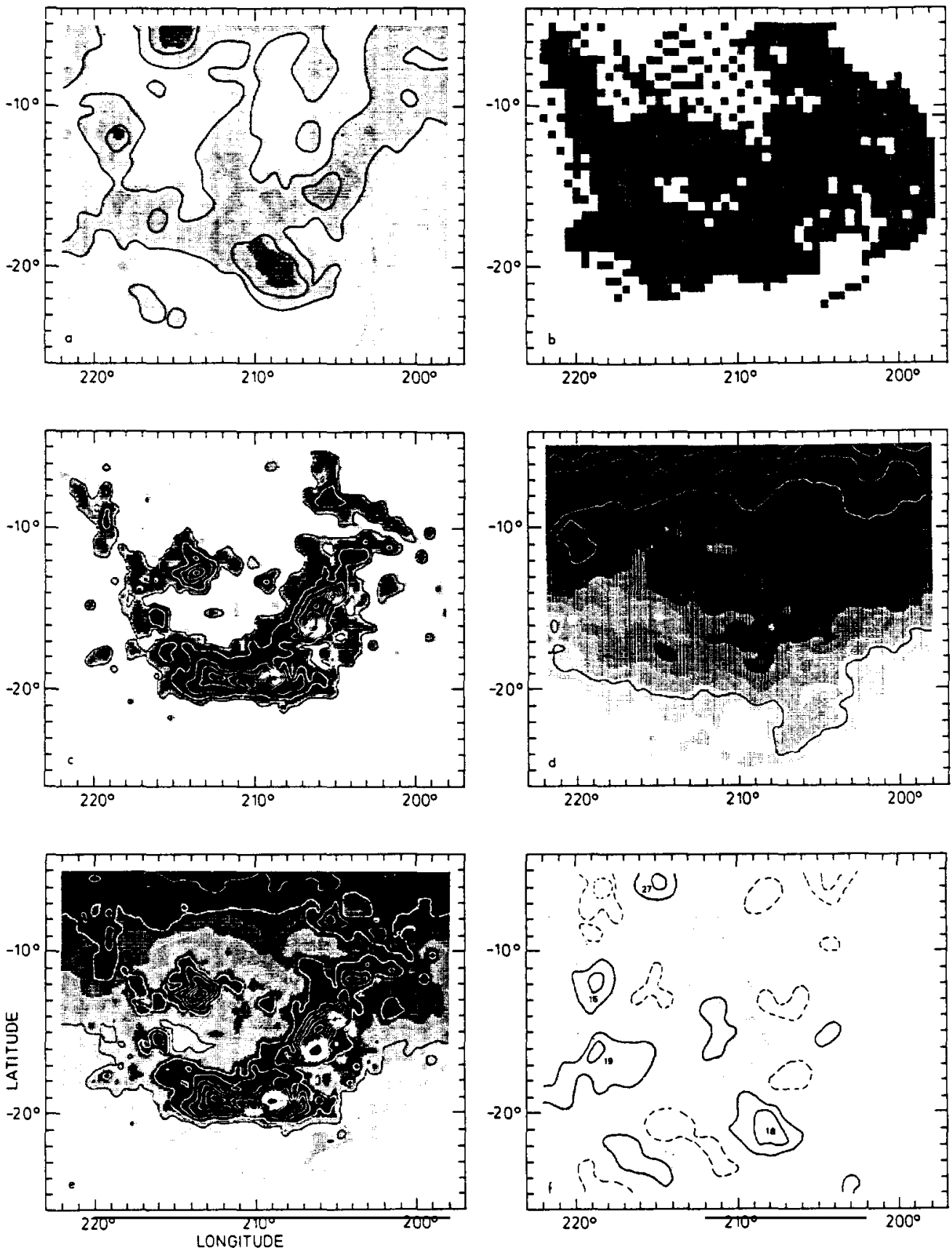

Fig. 1a-f. Gamma rays and the distribution of gas in the ori-Mon cloud complex. a. COS-B gamma-ray intensities in the 100-5000 MeV energy range, including the isotropic

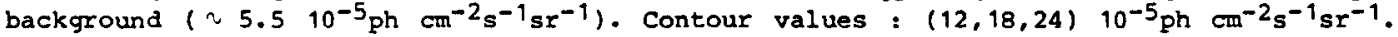
b. Coverage of the Columbia $C O$ survey. Each bin of $0.5^{\circ} \times 0.5^{\circ}$ that contains at least one observation is dark. $c$. Integrated intensities $w_{C 0}$ of $C O$ line emission at $2.6 \mathrm{~mm}$, smoothed to an angular resolution of $1 / 2^{\circ}$. Contour values $:(1,5,10,15 \ldots) \mathrm{K} \mathrm{km} \mathrm{s}^{-1}$. d. Total HI column-densities. Contour values : $(1.5,2.5,3.5, \ldots) 10^{20} \mathrm{H}$ atoms $\mathrm{cm}^{-2}$. e. Total gas column densities determined from $\mathrm{w}_{\mathrm{CO}}$ (Fig. c) and $\mathrm{N}(\mathrm{HI})$ (Fig.d), using $\mathrm{N}\left(\mathrm{H}_{2}\right) / \mathrm{w}_{\mathrm{CO}}=310^{20}$ molecules $\mathrm{cm}^{-2} \mathrm{~K}^{-1} \mathrm{~km}^{-1} \mathrm{~s}$ as found in the present analysis. Contour values : $(2,4,6, \ldots)$ ${ }_{10} 20_{\mathrm{H}}$ atoms $\mathrm{cm}^{-2}$. F. Map of the difference between the observed and the expected intensity distributions for the 100-5000 MeV energy range. The grey areas and the full contours indicate positive excesses in the subtracted map; the white regions and the dashed contours indicate deficiencies in the observed gamma-ray map. Contour values :

$(-8,-4,+4,+8) 10^{-5} \mathrm{ph} \mathrm{cm} \mathrm{cm}^{-2} \mathrm{~s}^{-1} \mathrm{sr}^{-1}$. The number of counts attributed to the excesses by a likelihood ratio test is indicated for excesses containing more than 10 counts. 

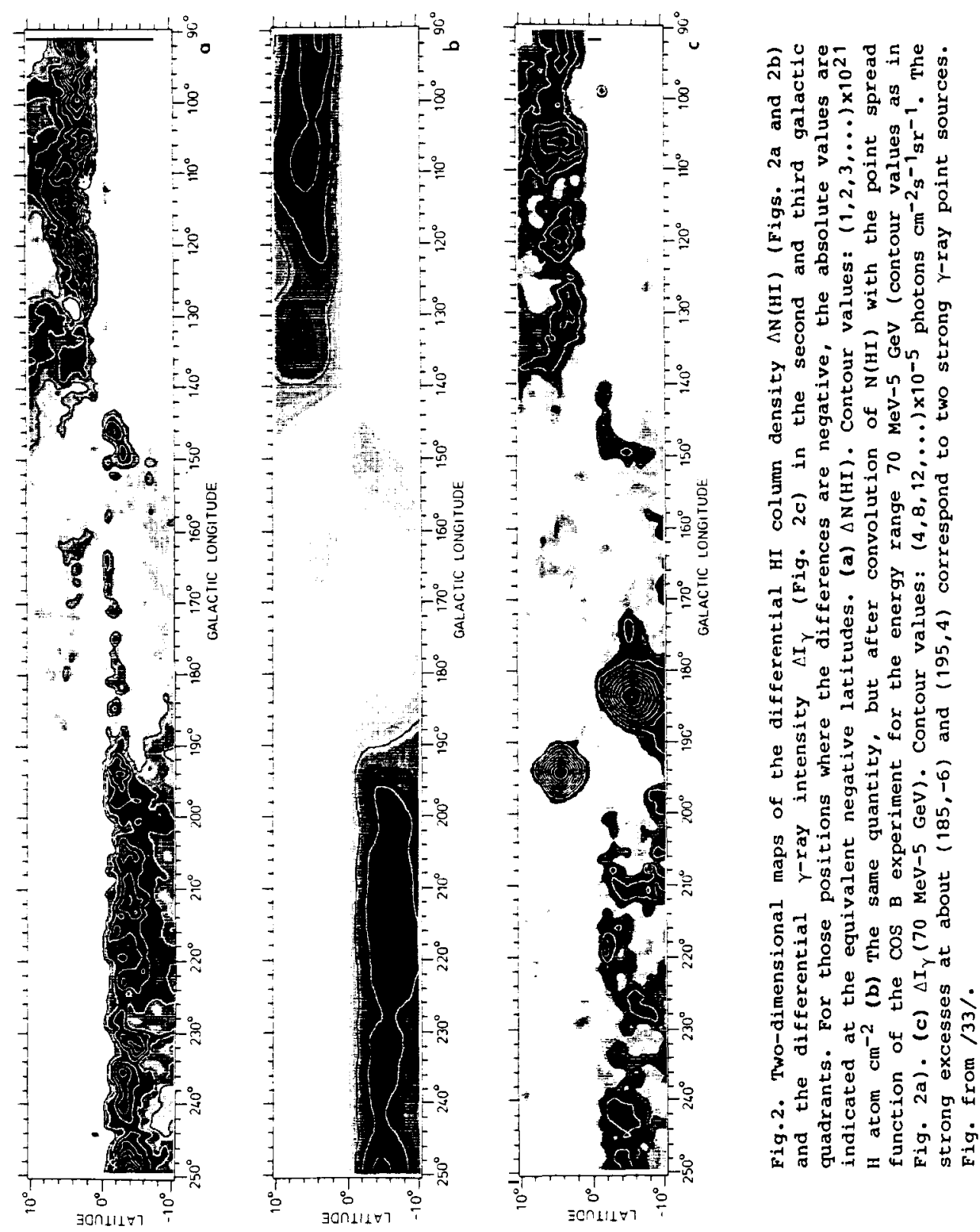

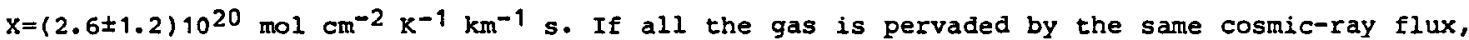
this value represents the average ratio $\left.\mathrm{N}_{2} \mathrm{H}_{2}\right) / \mathrm{w}_{\mathrm{CO}}$.

The 100-5000 MeV energy range was selected as a compromise between counting statistics and angular resolution (FWHM $\simeq 2.4^{\circ}$, HPBW $\simeq 4.3^{\circ}$ ), increasing the number of gamma counts that can be used in the analysis by a factor of about three compared to the 300-5000 MeV range. Since after convolution with the wider point-spread function for this energy range the smoothed distribution of $\mathrm{N}$ (HI) resembles closely the flat background over the largest part of the map, a three parameter $f i t$ is not allowed. Instead of treating $q_{y}$ as a free parameter, we used the average local emisivity, as determined from anlyses in other regions of the sky /15, 27/. This approach is justified since the gamma-ray emissivity found toward orion for the 70-5000 Mev energy range (using galaxy counts to trace the gas) /25/ and the value derived above for the 300-5000 MeV range are consistent with the local values.

The maximum-likelihood estimate of $I_{b}$ is again in agreement with previous estimates of the instrumental background level in this energy range, and the value for $x$ was found to be

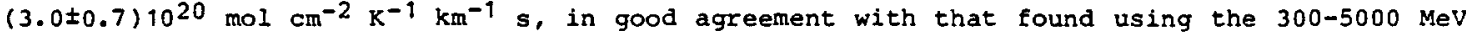
data.

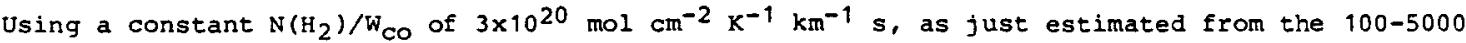



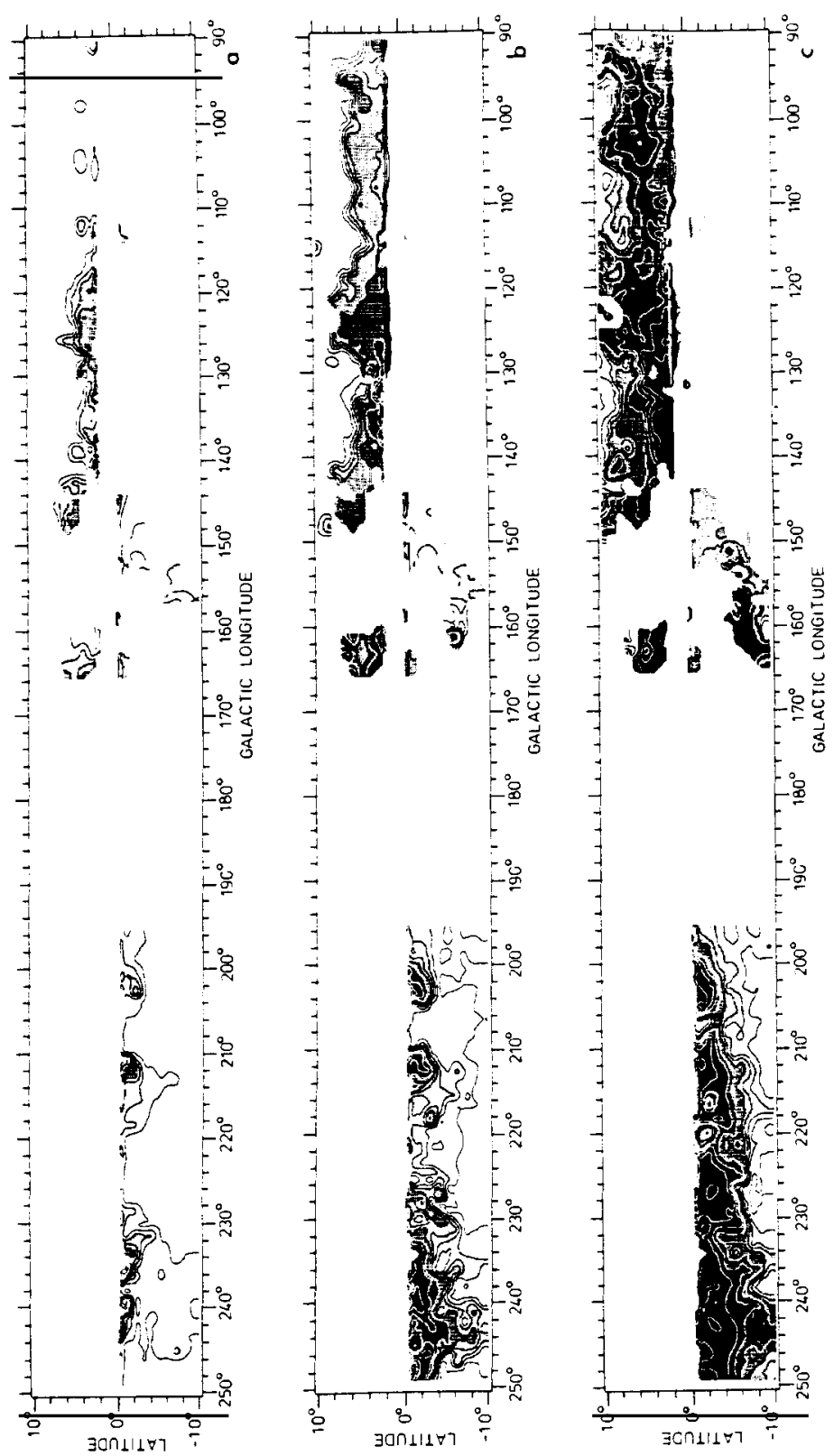

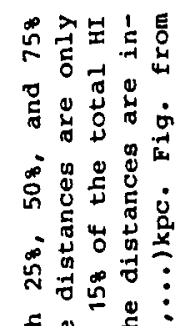

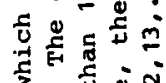

${ }^{3}$ ए

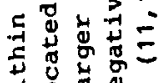

了过告

on

要画。

品

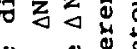

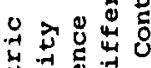

范

ठู

可

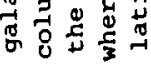

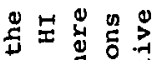

प

击明击

震出苛出

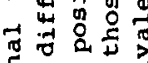

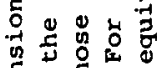

焉岩部

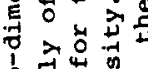

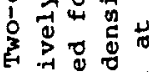

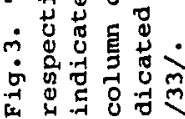

MeV gamma rays: Figure le shows the distribution of the total-gas column density as derived from the distributions of $\mathrm{HI}$ and $\mathrm{CO}$. Comparison with the gamma-ray (100-5000 MeV) map in Figure la shows that the total-gas distribution agrees well with the gama-ray distribution when the lower angular resolution of the COS-B map is taken into account. Figure if presents a map of the differences between the observed and expected intensity distributions. The number of counts attributed to the excesses by a likelihood ratio test $/ 30,31 /$ are indicated. No significant excesses above the expected diffuse radiation were found. It was also verified that none of the gamma-ray deficiencies are significant discrepancies from the gas estimate. In fact, all of the weak apparent excesses in Figure if are located at the edge of the CO coverage and are in part due to poor or no coverage of the co observations (compare with Figure $(b)$. Full details of the analysis and a discussion on the derived value for $x$ are given by Bloemen et al. $/ 32 \%$

RADIAL DISTRIBUTION OF COSMIC RAYS IN THE OUTER GALAXY

Extensive millimetre-wave surveys covering the whole outer galaxy are not yet completed. However, for this region Bloemen, Blitz and Hermsen /33/ have shown that the garma-ray intensity is proportional to the HI column density alone to within the uncertainty of the analysis (the $\mathrm{H}_{2}$ mass at $\mathrm{R}>\mathrm{R}_{0}$ they found to be $<3 \times 10^{8} \mathrm{M}_{\odot}$ ). Therefore the $\mathrm{w}_{\mathrm{co}}$ term can be deleted from equation (2) and $q_{y}$ (averaged over the entire line of sight) and $I_{b} c a n$ be determined fitting the gamma-ray and $\mathrm{N}$ (HI) distributions. 

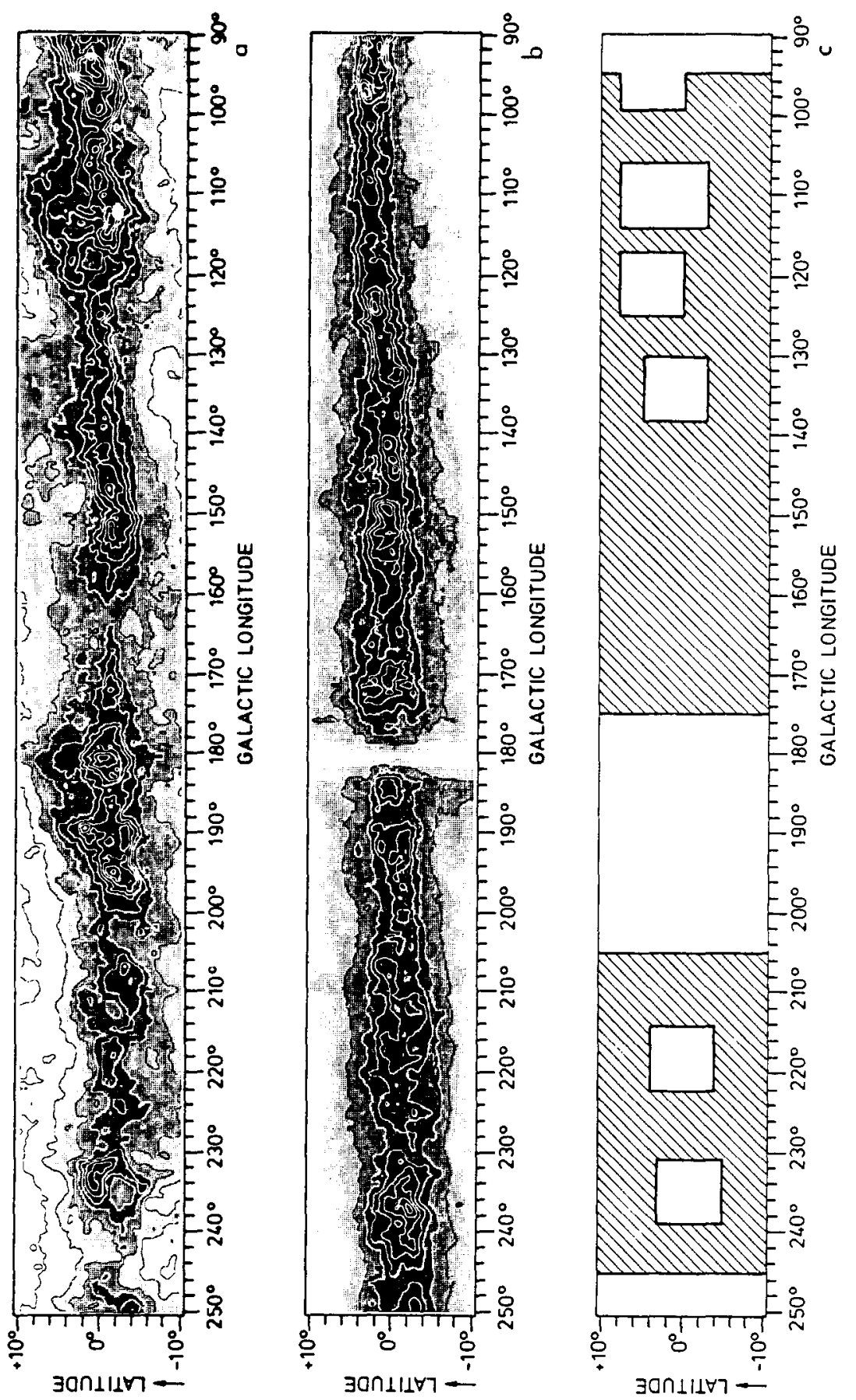

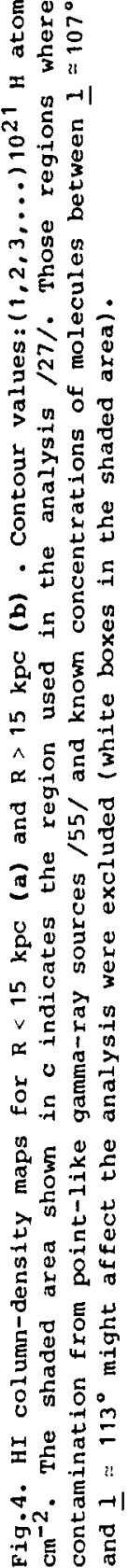

In $/ 33 /$ it was proven for the first time that the diffuse component of the galactic highenergy gamma-ray emission, and consequently the cosmic-ray particle distribution, extends to very large galacto-centric distances $(R \simeq 17 \mathrm{kpc})$. This could nicely be shown pictorially, exploiting the large-scale warp of the hydrogen layer $(\mathrm{e} . \mathrm{g} \cdot / 3 \dot{4}, 35 /)$. In addition, the analysis could be made independent of possible uncertainties in the mainly instrumental background level $I_{b}$. This was done by subtracting the data at negative latitudes from those at equivalent positive latitudes to provide a comparison between $\Delta I_{\gamma}$ and $\Delta N(H I)$, where $\Delta$ is the excess emission at positive latitudes. Figure 2 presents the two-dimensional maps of $\Delta I$ and $\Delta N(H I)$, showing the good detailed correlation between these quantities, thus indicating that the gamma-ray distribution must be strongly coupled to the HI distribution. Then, the velocity information available from the $21-\mathrm{cm}$ line can be used in combination with a rotation curve of the outer galaxy $/ 36,34$ / to determine the distances beyond the solar circle of the $\Delta N$ (HI) structures in Figure $21, \mathrm{~b}$. Figure 3 presents this distance information, indicating that an important fraction of $\Delta N(H I)$ is located at distances up to $R \sim 17 \mathrm{kpc}$, and therefore also the gamma-ray emissivity coupled to these distant structures originates from those large galactocentric distances. 


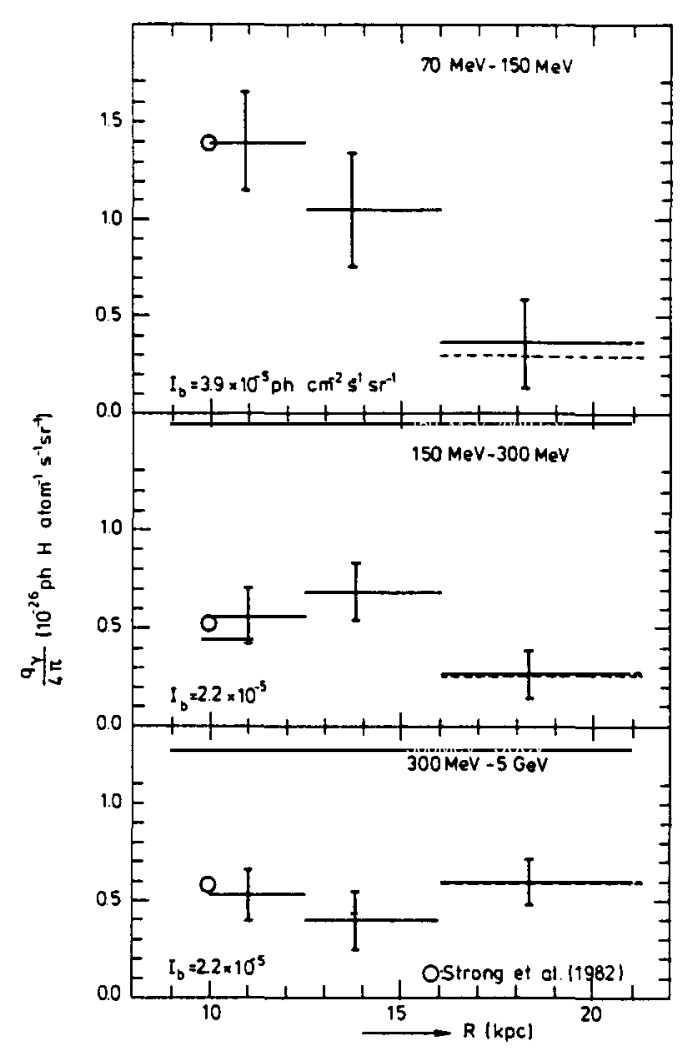

Fig.5. Radial distribution of $q_{y}$ in the outer Galaxy for three energy ranges. The resulting isotropic background levels $I_{b}$ are given in the figures.

The errorbars indicate formal 10 errors. The dashed lines for $R>16 \mathrm{kpc}$ show the values of $\mathrm{q}_{\gamma}$ after correction for a $\pi^{\circ}$-decay input spectrum.

\section{The Radial Distribution of Gamma-ray Emissivities}

In Bloemen et al. $/ 27 /$ the kinematics of HI have been used similarly to construct columndensity maps in various galacto-centric distance ranges in the outer galaxy. Figure 4 gives two examples of such $\mathrm{N}(\mathrm{HI})$ maps, namely for $10 \mathrm{kpc}<\mathrm{R}<15 \mathrm{kpc}$ and $\mathrm{R}>15 \mathrm{kpc}$. It is evident that the $\mathrm{N}(\mathrm{HI})$ distributions in these distance ranges are significantly different.

These differences remain after convolution with the energy dependent cos-B point-spread function for the three energy intervals $70-150 \mathrm{MeV}, 150-300 \mathrm{Mev}$ and 300-5000 Mev. Therefore, these maps can be used in combination with $\cos -B$ gamma-ray data to determine gamma-ray emissivities in these distance ranges using again a maximum likelihood method. In this case equation (2) should be replaced by a relation of the form :

$$
I_{\gamma}=\frac{1}{4 \pi} q_{\gamma,<15^{N}(H I)}<15+\frac{1}{4 \pi} q_{\gamma,>15^{N}(H I)}>15+I_{b},
$$

where $\mathrm{N}(\mathrm{HI})<15$ and $\mathrm{N}(\mathrm{HI})>15$ represent the $\mathrm{HI}$ column densities in the two distance ranges convolved with the gamma-ray point-spread function.

The analysis was performed over the sky area, shown in Figure 4c,covered by the Berkeley HI surveys, and in the three energy ranges given above. HI column-density maps have been constructed for the gas in three distance intervals : $R<12.5 \mathrm{kpc}, 12.5 \mathrm{kpc}<R<16 \mathrm{kpc}$ and $R>16$ kpc. A 4-parameter fit, using the maximum-likelihood analysis on $1^{\circ} \times 1^{\circ}$ bins, was applied in each energy interval. The resulting gamma-ray emissivities and background values are given for each energy range in Figure 5. The decrease in emissivity for the $70-150 \mathrm{MeV}$ energy range as a function of galacto-centric radius and the near constancy (within 208) for high energies $(300-5000 \mathrm{MeV})$, is evident from this figure. For the $70-150 \mathrm{MeV}$ range the likelihood was found to reduce by a factor of $\sim 10$ when a constant emissivity is assumed. The local emissivities from /15/ are included in Figure 5 for $R=10 \mathrm{kpc}$ and they fit the trend present in each energy range.

Figure 6 presents the gamma-ray emissivity spectrum for the distance ranges. There is a clear hardening of the spectrum for increasing galacto-centric distances outside the solar circle. The best power-law fits are indicated in the figure. For $R>16 \mathrm{kpc}$, the spectrum is equally well fitted by a $\pi^{\circ}$-decay spectrum and is thus the first measurement of a diffuse gamma-ray spectrum consistent with the $\pi^{\circ}$-decay spectrum. 


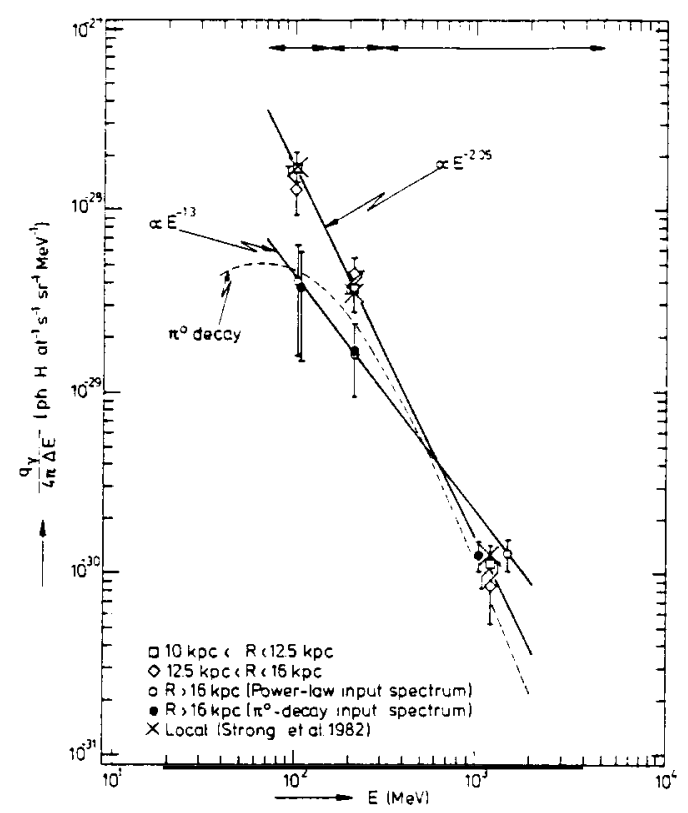

Fig.6. The gama-ray emissivity spectrum for three distance ranges in the outer Galaxy. Formal $(1 \sigma)$ errorbars are indicated. The energy ranges in which the emissivities are derived, are given at the top of the figure. The solid lines indicate the best power-law fits for $R<12.5 \mathrm{kpc}$ and $R>16 \mathrm{kpc}$. The dashed curve shows a $\pi^{\circ}$-decay spectrum $/ 29 /$.

\section{The Radial Distribution of Cosmic-ray Electrons and Nuclei}

The knowledge of the radial distribution of gamma-ray emissivities for different energy ranges enables the determination of the radial distribution of cosmic-ray electrons and protons separately, assuming that cosmic-ray-matter interactions are responsible for the observed gamma-ray emission. The inverse Compton contribution is most probably negligible, especially for the outer Galaxy (see e.g. $/ 37,38,39 /$ ).

The overall (power-law) shape of the local gamma-ray emissivity spectrum indicates that the dominant contribution to the low-energy component is due to electron bremsstrahlung. Also Stephens and Badhwar $129 /$ starting from the work of stecker $/ 28 /$, predicted for $\pi{ }^{\circ}$-decay from the demodulated spectrum of cosmic-ray nuclei a gama-ray emissivity in the $70-150$ Mev range of only $0.4410^{-26} \mathrm{ph} \mathrm{H}$ at $-1 \mathrm{~s}^{-1} \mathrm{sr}^{-1}$, which is 308 of the emissivity estimated from the observed local gamma rays (see e.g. /15/). For the 70-150 MeV range, about 80\% of the bremsstrahlung contribution is due to electrons with $E \approx 300 \mathrm{MeV}$. We have shown in Figure 5 that the low-energy gamu-ray component falls off rapidly with $R$, which implies that there is an evident galacto-centric gradient in the cosmic-ray electron density for electrons with $E \bumpeq 300 \mathrm{MeV}$, as has been known for some years. For the 300-5000 MeV range, about 808 of the small bremsstrahlung contribution is due to electrons with $\mathrm{E} \hat{\mathrm{GGeV}}$. It is reasonable to suppose that a similar decrease occurs in this higher-energy electron distribution. In fact, the cosmic-ray lifetime falls with increasing energy. Thus the gradient with $R$ could even be somewhat larger for higher electron energies. Since there is no detectable gradient for the high-energy gamma-ray emissivity, interactions due to the nuclear component of cosmic rays must dominate the gamma-ray spectrum at these energies. The absence of a gradient in the emissivities of the high-energy gama rays therefore implies that the density of cosmic-ray nuclei, with energies of a few GeV (those responsible for the bulk of the $\pi^{\circ}$-decay contribution in the 300-5000 MeV gamma-ray energy range), is constant to within our uncertainties out to large galacto-centric distances. This is supported by the close agreement between the measured gamma-ray emissivity for all distance ranges in the 300-5000 MeV range and that predicted for $\pi^{\circ}$-decay from the demodulated spectrum of cosmic-ray nuclei $\left(0.4510^{-26}\right.$ ph $\left.\mathrm{H} \mathrm{at} \mathrm{t}^{-1} \mathrm{~s}^{-1} \mathrm{sr}^{-1}\right) / 29 /$.

If we assume that the electron density decreases linearly with $R$ and that the electron densi$t y$ is effectively zero for $R 18 \mathrm{kpc}$ (see Figure 5), the radial distribution of the cosmic-ray electron density $n_{e}(R)$ and of the nuclear density $n_{n}(R)$ in the outer Galaxy is described by:

$$
n_{e}(R)=n_{e}\left(2.25-1.25 \text {, with } n_{e}(R)=0 \text { for } R>18 \mathrm{kpc} \text {, and } n_{n}(R)=n_{n}\right. \text {, }
$$

where $n_{e}$ and $n_{n}$ are the local interstellar electron and nuclear densities respectively. The variation of the electron component is consistent with results based on low-frequency radio continuum surveys (e.g. at $30 \mathrm{MHz} / 40 /$ and at $408 \mathrm{MHz} / 41 /$ ). 
In the inner galaxy molecular hydrogen is an important constituent of the total interstellar gas, although the relative contribution of $\mathrm{H}_{2}$ is still an open question (see, e.g. $/ 17 /$ ). Since only in the first galactic quadrant a fully-sampled co survey over a large latitude range $/ 10 /$ is available, a correlation study between gamma-ray data and total-gas data is only possible in that longitude range $142,43 /$.

A detailed maximum-likelihood analysis using equation (2) has been performed by Lebrun et al. $/ 43 /$, exploiting the $\cos -B$ data base. As a first step they limited their study to the higherergy gamma rays (E $>300 \mathrm{MeV})$, taking advantage of the relatively good angular resolution at these energies. In addition, the contribution of inverse-Compton gamma rays in this energy range is expected to be negligible $/ 37,38,39 \%$. It is found that a simple model, in which uniformly distributed cosmic rays interact with the interstellar gas, as traced by $\mathrm{HI}$ and Co,can account for almost all the observed gamma rays. Furthermore, if the contribution frorn point sources to the gamna-ray flux is significant, these sources must have a galactic distribution similar to that of $\mathrm{CO}$. In fact, in a follow-up paper $/ 44 / \mathrm{studying}$ the same data bases in a search for gamma-ray sources, it is shown that 5 ganma-ray excesses in the first galactic quadrant are not explained by the gas distribution and an average cosmic-ray density. From the maximum likelihood fit for the first galactic quadrant /43/ an average value $q_{Y}(300-5000 \mathrm{MeV})=0.55 \times 10^{-26} \mathrm{ph} \mathrm{H}$ at $^{-1} \mathrm{~s}^{-1} \mathrm{sr}^{-1}$ can be derived, indicating that any cosmic-ray yradient, predominantly cosmic-ray nuclei for this garma-ray energy range, in the inner galaxy is likely to be small, consistent with the distribution for the outer galaxy (see Figure 5 ).

The average gamma-ray spectra in the second and third galactic quadrants are harder than the average spectra in the first and fourth galactic quadrants (see e.g. $/ 45 /$ ). This could be the result of a continuous softening of the gamma-ray emission toward inner-galactic regions, i.e. an increase of the relative contribution from cosmic-ray electrons, as discussed by Hermsen and Bloemen $/ 46 /$. Such a gradient in the cosmic-ray electron distribution would be consistent with the large-scale galacti= synchrotron emissivity (see e.g. $/ 47 /$ ). It is evident that a stronger gradient in the cosmic-ray electron distribution than in the nuclei distribution towards the inner galaxy agrees with a smooth extrapolation into the Galaxy of the radial distribution derived above for the outer galaxy,

Also in other studies of the gamma-ray data (e.g. /48/) cosmic-ray gradients were shown to exist in the inner galaxy, but no differences were claimed between the electron and nuclei distributions. In most earlier attempts to explain the gamma-ray emissivity in the inner galaxy (e.g. also the recent Fichtel and Kniffen /49/ paper) more-detailed models (e.g. spiral arm models) of the interstellar gas and the cosmic-ray distributions are fitted to the gamma-ray intensity distribution. However, a more direct approach is now possible using in a maximum likelihood analysis in addition to equation (2) also the distance information in the kinematics of the $\mathrm{HI}$ and $\mathrm{CO}$. An analysis similar to that presented above for the outer galaxy will give direct information on the presence of gradients in the cosmic-ray particle distributions in the inner galaxy. This will be the subject of a forthcoming paper $/ 50 /$.

\section{COSMIC-RAY DENSITY AND TOTAL MASS IN THE GALACTIC CENTRE}

Astronomical studies of the central region of the Galaxy are in many respects intriguing. This is certainly also the case for the study of possible garma-ray emission from the galactic centre. In early attempts to interpret the gamma-ray distribution in the galactic centre region in combination with total-gas estimates the required cosmic-ray densities at the galactic centre differed by up to three orders of magnitude $/ 51,52,48 /$. These large differences were due to uncertainties in the ganma-ray data as well as large uncertainties in the mass estimates of the gas. The latter is still uncertain by at least an order of magnitude : Millimeter wave observers have argued that the $\mathrm{H}_{2}$ masses implied by their observations are typically $\gtrsim 3 \times 10^{8} \mathrm{M}_{0}$, but oort $/ 53 /$ has presented arguments for a mass in the galactic centre (referring to the central $400 \mathrm{pc}$ ) an order of magnitude lower.

In the Blitz et al./54/ paper cos-B gamma-ray data in the energy range 300-5000 MeV have been selected to construct with the best possible angular resolution a gamma-ray map covering the galactic centre and the neighbouring disk region. Over the same sky area the HI column density can be determined from $21-\mathrm{cm}$ surveys $/ 20,21 /$ and, more importantly, extensive co observations sampled every $0.5^{\circ}$ in longitude from $1=350^{\circ}$ to $1=25^{\circ}$ at latitudes $b=-20^{\prime},-10^{\prime}, 0^{\prime},+10^{\prime}$, and $+20^{\circ} / 22,23,24 /$ are available. Figure $7 \bar{a}$ shows the longitude distribution of $\mathrm{w}_{c o}$ averaged over $|b| \leqslant 20^{\prime}$. The latitude coverage of the co observations is narrower than the width of the cos-B point-spread function. In order to convolve the $C O$ observations with the cos- $B$ pointspread function, the $C O$ data are extra-polated by assuming a gaussian latitude distribution (see for details /54/). For the comparison of the gamma-ray distribution with the total-gas distribution following equation (2) also the N(HI) distribution is convolved, rendering a distribution which is approximately flat (within $\sim 15 \%$ ) as a function of longitude over the longitude range shown in Figure 7.

Using the values of the parameters in equation (2), $q_{\gamma}, X$ and $I_{b}$, determined from a maximum 

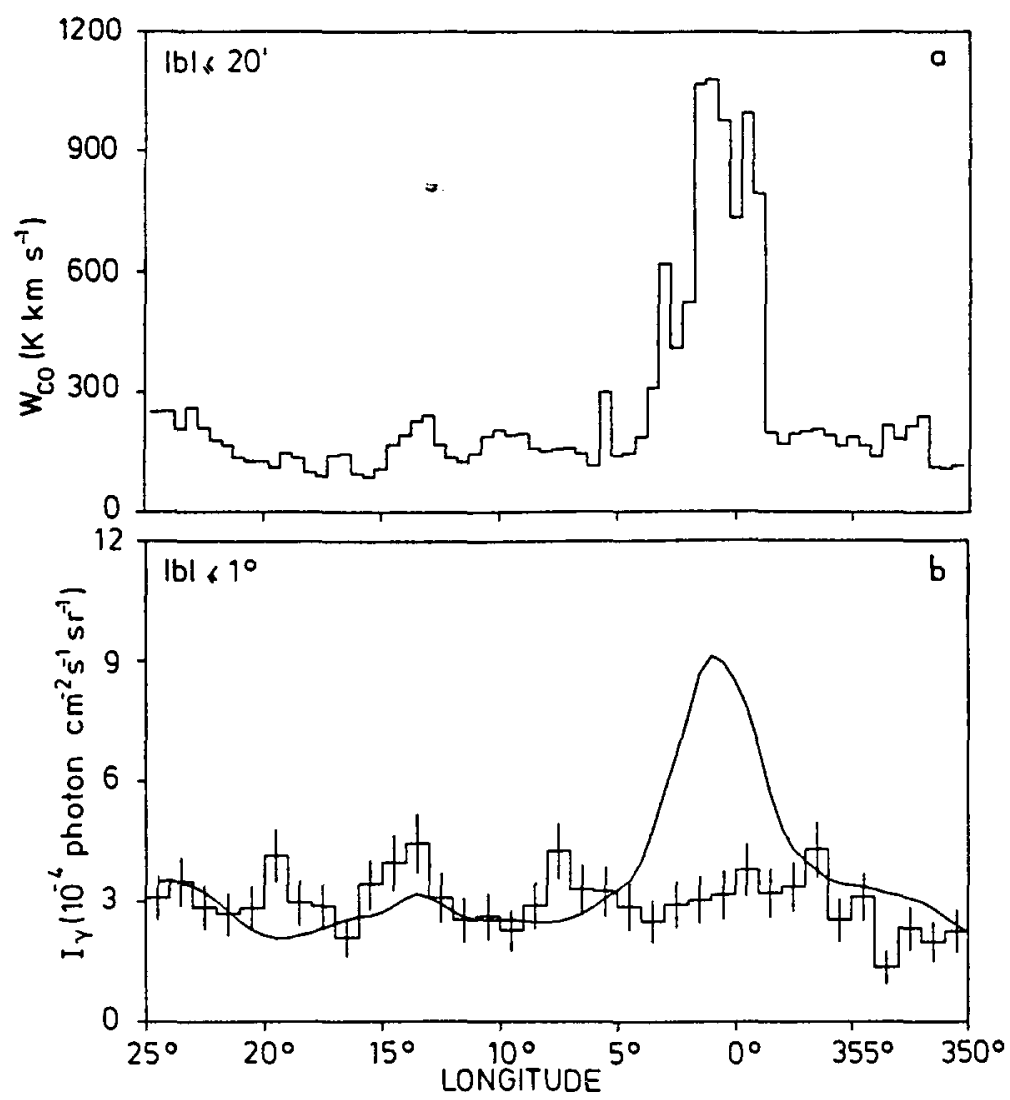

Fig.7a,b. Longitude distributions of : a. Carbon monoxide antenna temperature integrated over velocity and latitude for $-20^{\prime}<b<20^{\prime}$. b. The observed (histogram) and expected gamma-ray intensities $(300-5000 \mathrm{MeV})$ for $-1^{\circ}<b<1^{\circ}$. The solid line shows the expected gamma-ray intensities from $\mathrm{HI}$ and co data using $\varepsilon=0.55$ photon $\mathrm{cm}^{-2} \mathrm{H}$ at $\mathrm{s}^{-1} \mathrm{sr}^{-1}$ and $\mathrm{N}\left(\mathrm{H}_{2}\right) / \mathrm{W}_{\mathrm{co}}=3 \times 10^{20}$ molecules $\mathrm{cm}^{-2} \mathrm{~K}^{-1} \mathrm{~km}^{-1} \mathrm{~s}^{1}$. The small gamma-ray exesses near $1=356^{\circ}, 1=7^{\circ}$ and $1=14^{\circ}$ are close to three gamma-ray sources listed in the 2 CG catalogue $/ 18,55 \%$.

likelihood analysis for nearly all of the first quadrant (see above and /43/) a ganua-ray distribution covering the galactic centre can be predicted and compared with the actual measurements. Figure $7 \mathrm{~b}$ shows this comparison $/ 54 /$. Although the expected and observed gamma-ray intensities are in good agreement in the disk, the predicted peak toward the galactic centre is not observed. The $3 \sigma$ upper limit to any excess gama-ray flux within the range $|b|<3^{\circ} ; 357^{\circ}<1<5^{\circ}$, over that produced in the disk is $4 \times 10^{-7}$ photon (300-5000 MeV) $\mathrm{cm}^{-2} \mathrm{~s}^{-T}$. This result implies that in the galactic centre the gama-ray emissivity is anomalously low, or that molecular hydrogen is nearly an order of magnitude less abundant than estimates made from co observations. The former suggests that the density of cosmic-ray nuclei with energies of $\sim 1 \mathrm{GeV}$ is anomalously small relative to the local value, or perhaps that these cosmic rays do not efficiently penetrate the molecular clouds in the central region of the Galaxy. The latter suggests that the gas-to-dust ratio is anomalously low and that the $3 \sigma$ upper limit to the mass of the nuclear disk is $5.8 \times 10^{7} \mathrm{M}_{\odot}$. Circumstantial evidence favours a low $\mathrm{H}_{2} / \mathrm{O}$ abundance as the source of the gama-ray deficiency $/ 54 /$.

\section{Acknowledgements}

We thank our colleagues and co-authors of the various works summarized in this paper for many helpful discussions. The Laboratory for Space Research Leiden is supported financially by the Netherlands Organisation for the Advancement of Pure Research (ZwO).

\section{REFERENCES}

1. W. Baade, and F. Zwicky, Phys. Rev. 45, 138 (1934)

2. G.R. Blumenthal, and R.J. Gould, Rev. Mod. Phys. 42, 237 (1970)

3. G.F. Bignami, C.E. Fichtel, D.A. Kniffen, and D.J. Thompson, Ap.J. 199, 54 (1975) 
4. F.W. Stecker, P.M. Solomon, N.Z. Scoville, and C.E. Ryter, Ap.J. 201, 90 (1975)

5. J.A. Paul, M. Cassé, and C.J. Cesarsky, Ap.J. 207, 62 (1976)

6. C.J. Cesarsky, M. Cassé, and J.A. Paul, Astron. Astrophys. 60, 139 (1977)

7. J.C. Higdon, Ap.J. 232, 113 (1979)

8. M.L. Kutner, K.D. Tucker, G. Chin, and P. Thaddeus, Ap.J. 215, 521 (1977)

9. R.J. Maddalena, M. Morris, J. Moscowitz, and P. Thaddeus, private communication (1984)

10. T.M. Dame, and P. Thaddeus, private communication (1984)

11. L. Scarsi, K. Bennett, G.F. Bignami, G. Boella, R. Buccheri, W. Hermsen, L. Koch, H.A. Mayer-Hasselwander, J.A. Paul, E. Pfefferman, R. Stiglitz, B.N. Swanenburg, B.G. Taylor, and R.D. Wills, in : Recent Advances in Gamma-Ray Astronomy, eds. R.D. Wills, B. Battrick, ESA SP-124, 3 (1977)

12. H.A. Mayer-Hasselwander, X. Bennett, G.F. Bignami, R. Buccheri, P.A. Caraveo, W. Hermsen,G. Kanbach, F. Lebrun, G.G. Lichti, J.L. Masnou, J.A. Paul, K. Pinkau, B. Sacco, L. Scarsi, B.N. Swanenburg, and R.D. Wills, Astron. Astrophys. 105, 164 (1982)

13. C.E. Fichtel, R.C. Hartman, D.A. Kniffen, D.J. Thompson, G.F. Bignami, H. Ögelman, M.E. Özel, and T. Tümer, Ap.J. 198, 163 (1975)

14. F. Lebrun, G.F. Bignami, R. Buccheri, P.A. Caraveo, W. Hermsen, G. Kanbach, H.A. Mayer-Hasselwander, J.A. Paul, A.W. Strong, and R.D. Wills, Astron.Astrophys. 107, 390

15. A.W. Strong, G.F. Bignami, J.B.G.M. Bloemen, R. Buccheri, P.A. Caraveo, K. Hermsen, G. Kanbach, F. Lebrun, H.A. Mayer-Hasselwander, J.A. Paul and R.D. Wills, Astron. Astrophys. 115, 404 (1982)

16. F. Lebrun, and J.A. Paul, Ap.J. 266, 276 (1983)

17. J. Lequeux, Comments on Astrophysics 9, 117 (1981)

18. W. Hermsen, Ph.D. Thesis, University of Leiden, The Netherlands (1980)

19. C. Heiles, and H.J. Habing, Astron. Astrophys. Suppl. 14, 1 (1974)

20. H. Weaver, and D.R.W. Williams, Astron. Astrophys. Suppl. 8, 1 (1973)

21. A.W. Strong, P.A. Riley, J.L. Osborne, and J.D. Murray, MNRAS 201, 495 (1982)

22. T.M. Banta, Ap.J. 216, 381 (1977)

23. T.M. Banla, Ap.J. 242, $95(1980)$

24. T.M. Bania, private comunication (1984)

25. P.A. Caraveo, K. Bennett, G.F. Bignami, W. Hermsen, G. Kanbach, F. Lebrun, J.L. Masnou, H.A. Mayer-Hasselwander, J.A. Paul, B. Sacco, L. Scarsi, A.W. Strong, B.N. Swanenburg, and R.D. Wills, Astron. Astrophys. 91, L3 (1980)

26. P.A. Caraveo, I. Barbareschi, K. Bennett, G.F. Bignami, W. Hermsen, G. Kanbach, F. Lebrun, J.L. Masnou, H.A. Mayer-Hasselwander, B. Sacco, A.W. Strong, and R.D. Wills, in Proc. 17th Int. Cosmic Ray Conf., Paris, 1, p. 139 (1981)

27. J.B.G.M. Bloemen, K. Bennett, G.F. Bignami, L. Blitz, P.A. Caraveo, M. Gottwald, W. Hermsen, F. Lebrun, H.A. Mayer-Hasselwander, and A.W Strong Astron. Astrophys. 135, 12 (1984)

28. F.W. Stecker, Astrophys. Space Sci. 6, 377 (1970)

29. S.A. Stephens, G.D. Badhwar, Astrophys. Space Sci. 76, 213 (1981)

30. W. Cash, Ap.J. 228, 939 (1979)

31. A.M.T. Pollock, K. Bennett, G.F. Bignami, J.B.G.M. Bloemen, R. Buccheri, P.A. Caraveo, W. Hermsen, G. Kanbach, F. Lebrun, H.A. Mayer-Hassel wander, and A.W. Strong, submitted to Astron. Astrophys. (1984) 
32. J.B.G.M. Bloemen, P.A. Caraveo, W. Hermsen, F. Lebrun, R.J. Maddalena, A.W. Strong, and P. Thaddeus, Astron. Astrophys., in press (1984)

33. J.B.G.M. Bloemen, L. Blitz, and W. Hermsen, Ap.J. 279, 136 (1984)

34. S.R. Kulkarni, L. Blitz, and C. Heiles, Ap.J (Letter) 259, L63 (1982)

35. A.P. Henderson, P.D. Jackson, and F.J. Kerr, Ap.J. 263, 116 (1982)

36. L. Blitz, M. Fich, and A.A. Stark, Ap. J. Suppl. 49, 183 (1982)

37. D.A. Kniffen, and C.E. Fichtel, Ap.J., 250, 389 (1981)

38. W. Sacher, and V. Schönfelder, Space Sci. Rev. 36, 249 (1983)

39. J.B.G.M. Bloemen, submitted to Astron. Astrophys. (1984)

40. W.R. Webber, G.A. Simpson, and H.V. Cane, Ap.J. 236, 448 (1980)

41. S. Phillips, S. Kearsey, J.L. Osborne, C.G.T. Haslam, H. Stoffel, Astron. Astrophys. 98, $286(1981)$

42. K. Arnaud, L.T. Pei, P.A. Riley, A.W. Wolfendale, T.M. Dame, J.E. Brock, P. Thaddeus, MNRAS 194, IP (1982)

43. F. Lebrun, K. Bennett, G.F. Bignami, J.B.G.M. Bloemen, R. Buccheri, P.A. Caraveo, M. Gottwald, W. Hermsen, G. Kanbach, H.A. Mayer-Haselwander, T. Montmerle, J.A. Paul, B. Sacco, A.W. Strong, and R.D. Wills, Ap.J. 274, 231 (1983)

44. A.M.T. Pollock, K. Bennett, G.F. Bignami, J.B.G.M. Bloemen, R. Buccheri, P.A. Caraveo,W. Hermsen, G. Kanbach, F. Lebrun, H.A. Mayer-Hasselwander, and A.W. Strong, submitted to Astron.Astrophys. (1984)

45. H.A. Mayer-Hasselwander, in : Kinematics, Dynamics and Structure of the Milky Way, ed. W.L.H. Shuter, D. Reidel Publ. Comp. Dordrecht, Holland 1983, p.223.

46. W. Hermsen, and J.B.G.M. Bloemen, in: Surveys of the Southern Galaxy, eds. W.B. Burton, F.P. Israel, D. Reidel Publ. Comp. Dordrecht, Holland 1983, p.65

47 R.L. Brown, in: Kinematics, Dynamics and Structure of the Milky Way, ed. W.I.H. Shuter, D. Reidel Publ. Comp. Dordrecht, Holland 1983, P. 197.

48. M.R. Issa, P.A. Riley, A.W. Strong, and A.W. Wolfendale, J. Phys. G7, 973 (1981)

49. C.E. Fichtel, and D.A. Kniffen, Astron. Astrophys. 134, 13 (1984)

50. J.B.G.M. Bloemen et al., Caravane Collaboration for the cos-B satellite, private comunication (1984)

51. A.W. Wolfendale, and D. Worral, Nature 263,482 (1977)

52. J. Audouze, J. Lequeux, J.-L. Masnou, and J.-L. Puget, Astron. Astrophys. 80,276 (1979)

53. J.H. Oort, Ann. Rev. Astron. Astrophys. 15, 295 (1977)

54. I. Blitz, J.B.G.M. Bloemen, w. Hermsen, T.M. Bania, Astron. Astrophys. in press (1984)

55. B.N. Swanenburg, K. Bennett, G.F. Bignami, R. Buccheri, P.A. Caraveo, W. Hermsen, G. Kanbach, G.G. Lichti, J.L. Masnou, H.A. Mayer-Hasselwander, J.A. Paul, B. Sacco, I. Scarsi, and R.D. Wills, Ap.J. (Letters) 243, L69 (1981) 